\title{
Phenotype variations in Lafora progressive myoclonus epilepsy: possible involvement of genetic modifiers?
}

\author{
Shweta Singh $^{1}$ and Subramaniam Ganesh ${ }^{2}$ \\ Lafora progressive myoclonus epilepsy, also known as Lafora disease (LD), is the most severe and fatal form of progressive \\ myoclonus epilepsy with its typical onset during the late childhood or early adolescence. LD is characterized by recurrent \\ epileptic seizures and progressive decline in intellectual function. LD can be caused by defects in any of the two known genes \\ and the clinical features of these two genetic groups are almost identical. The past one decade has witnessed considerable \\ success in identifying the $L D$ genes, their mutations, the cellular functions of gene products and on molecular basis of LD. \\ Here, we briefly review the current literature on the phenotype variations, on possible presence of genetic modifiers, and \\ candidate modifiers as targets for therapeutic interventions in LD.
}

Journal of Human Genetics (2012) 57, 283-285; doi:10.1038/jhg.2012.29; published online 29 March 2012

Keywords: epilepsy; genetic modifiers; glycogen metabolism; Mendelian disorders; neurodegeneration; protein-protein interaction

Progressive myoclonus epilepsies (PMEs) are a group of rare genetic disorders characterized by myoclonic seizures and progressive neurological deterioration. ${ }^{1}$ Among the progressive myoclonus epilepsies, Lafora type epilepsy, also known as Lafora disease (LD), is unique because clinical variations are not that common, yet the disease can be caused by defects in any of the three chromosomal loci (6q24, 6p22.3 and one unknown locus). ${ }^{1-4}$ Two genes for LD have already been identified, ${ }^{3}$ and evidences for the presence of a third locus are ample. ${ }^{3,5}$ Clinical course of the LD is characterized by the onset of myoclonus, grand mal, tonic clonic and absence seizures during the adolescence or in the late childhood. Cognitive decline, dystharthia and ataxia are noted at 11-17 years. ${ }^{1}$ Patients are usually bedridden at around 20 years and then on survival requires respiratory assistance. ${ }^{1,6}$ Pathologically, LD is characterized by the presence of pathognomonic intra-neuronal cytoplasmic inclusions called the Lafora bodies. ${ }^{4,6}$ These inclusions stain positive for the periodic acid-Schiff reagent, suggesting the presence of a large amount of carbohydrate. Biochemical studies demonstrate that these inclusions are made up of abnormally lesser branched and hyperphosphorylated glycogenmoieties. ${ }^{7,8}$ Besides the Lafora bodies, neurodegenerative changes are also seen. ${ }^{9}$ Nonetheless, the cause and consequence of Lafora bodies in LD pathogenesis are yet to be unequivocally established.

The two genes known for LD have been extensively characterized. These are the EPM2A and NHLRC1 genes, coding for a protein phosphatase (named laforin) and an E3 ubiquitin ligase (named malin), respectively. ${ }^{3}$ Laforin and malin interact with each other and as a complex participate in diverse cellular pathways, including the glycogen metabolism, ubiquitin-proteasome system and in heat shock response, ${ }^{3,10-13}$ and therefore defects in these processes are thought underlie LD. ${ }^{3}$ A number of disease-causing mutations, more than 100 of them, are known in EPM2A and NHLRC1, and several studies have looked at possible genotype-phenotype correlations in the LD families. ${ }^{3}$ The general consensus is that it would be difficult to 'predict' the defective gene during the early stages of LD. ${ }^{3}$ Indeed, no significant difference in the clinical symptoms was observed in patients harboring mutations in the EPM2A gene as compared with those who have had mutations in the NHLRC1 gene, although the NHLRC1 patients tend to live longer than the other group. ${ }^{14,15}$ Therefore, it was suggested that malin is one of the upstream regulators of laforin and therefore mutations in malin would affect a few but not all functions of laforin, thus resulting in a milder course of disease. ${ }^{15}$ Similarly, an early onset LD phenotype was observed for the EPM2A patients with defects affecting the exon-1 of the gene. ${ }^{6,16}$ Here it was suggested that defects in the amino-terminal carbohydrate-binding domain of laforin, mainly coded by the first exon of the EPM2A gene, might underlie the early onset phenotype. ${ }^{6}$ Although the proposed models were attractive, subsequent studies have shown that such genotype-phenotype correlations cannot be extended to several other group of patients, ${ }^{17,18}$ suggesting that these models, at best, could be applicable to a few LD families. For examples, several of the NHLRC1 patients studied showed severe form $\mathrm{LD}^{19}$ or a late onset $\mathrm{LD},{ }^{20}$ and not all EPM2A patients with exon 1 mutations showed an early onset of the LD symptoms. ${ }^{17,18}$ Intriguingly, a founder effect mutation for EPM2A in Arab families showed variable age at onset for $\mathrm{LD},{ }^{21}$ suggesting that a specific

${ }^{1}$ Department of Human Biology, International Medical University, Kuala Lumpur, Malaysia and ${ }^{2}$ Department of Biological Sciences and Bioengineering, Indian Institute of Technology, Kanpur, India

Correspondence: Dr S Singh, Department of Human Biology, International Medical University, No. 126, Jalan Jalil Perkasa 19, Bukit Jalil, Kuala Lumpur, WP 57000 , Malaysia. E-mail: shweta_singh@imu.edu.my

Received 19 January 2012; revised 20 February 2012; accepted 28 February 2012; published online 29 March 2012 
Table 1 Interacting partners of laforin and malin and their physiological functions

\begin{tabular}{|c|c|c|c|}
\hline Interacting partners & Interacts with & Proposed/established cellular functions of the partner & Reference \\
\hline HIRIP5 & Laforin & Laforin interacts with and dephosphorylate HIRIP5; functional significance unknown. & 34 \\
\hline EPM2AIP1 & Laforin & Interacts with laforin; functional significance unknown. & 35 \\
\hline Glycogen synthase kinase 3 (GSK3) & Laforin and malin & $\begin{array}{l}\text { Laforin and malin bind to GSK3, and laforin dephosphorylates GSK3; GSK3 is involved } \\
\text { in energy metabolism, neuronal cell development and body pattern formation. }\end{array}$ & 36,37 \\
\hline Glycogen-debranching enzyme (AGL) & Malin & Malin interacts with and promotes the ubiquitination of AGL. & 38 \\
\hline AMP-activated protein kinase (AMPK) & Laforin and malin & $\begin{array}{l}\text { Facilitates malin-mediated degradation of laforin by interacting with and phosphorylating } \\
\text { laforin; malin interacts with and ubiqutinate AMPK subunit beta to modulate some } \\
\text { unknown function. }\end{array}$ & $10,13,39$ \\
\hline Protein targeting to glycogen (PTG/R5) & Laforin-malin complex & $\begin{array}{l}\text { PTG is a scaffold protein that binds to glycogen and many of the enzymes and promote } \\
\text { glycogen synthesis and the level of PTG is regulated by the malin-laforin complex }\end{array}$ & $10,24,40,41$ \\
\hline Heat shock protein 70 (HSP70) & Laforin-malin complex & $\begin{array}{l}\text { Laforin, malin and Hsp70 (a cellular chaperone) as a non-redundant partners suppress } \\
\text { the cellular toxicity of misfolded proteins. }\end{array}$ & 11 \\
\hline Tau protein & Laforin & Laforin interacts with and dephosphorylate tau, a microtubule-binding protein. & 42 \\
\hline Heat shock factor-1 (HSF1) & Laforin-malin complex & $\begin{array}{l}\text { Laforin and malin contribute to the activation HSF1-the heat shock-dependent } \\
\text { transcription factor, during stress by an unknown mechanism. }\end{array}$ & 12 \\
\hline $\begin{array}{l}\text { Carboxyl terminus of Hsp70-interacting } \\
\text { protein (CHIP) }\end{array}$ & Laforin-malin complex & $\begin{array}{l}\mathrm{CHIP} \text {, a co-chaperone, requires malin-laforin for its translocation to the nucleus upon } \\
\text { heat shock and to confer protection against heat stress. }\end{array}$ & 12,43 \\
\hline Neuronatin & Malin & $\begin{array}{l}\text { A proteolipid that regulates ion channels during brain development stimulates glycogen } \\
\text { synthesis in a malin-dependent way. }\end{array}$ & 44 \\
\hline Dishevelled2 & Malin & A key mediator of Wnt signaling pathway, is regulated by malin via its degradation & 45 \\
\hline
\end{tabular}

mutation per se may not underlie a subsyndrome in LD. Conversely, NHLRC1 patients from a genetic isolate of tribal Oman with the same mutation showed identical age at onset and similar clinical course of the disease. ${ }^{22}$ Thus, factors other than the defective $L D$ gene could possibly be involved in modifying the disease onset or the progression in some cases. Indeed this has been suggested in an LD family wherein one of the two affected patients presented severe liver failure at onset of $\mathrm{LD}$, although both siblings are homozygotes for the same EPM2A mutation. ${ }^{23}$

The $L D$ gene products laforin and malin are known to interact with a diverse set of proteins and (see Table 1), therefore, variations in gene(s) that code for these interacting protein(s) might contribute to variations in phenotype. Indeed, a recent study demonstrates that a sequence variant in the $P P P 1 R 3 C$ gene coding for the protein targeting to glycogen (PTG) contributes to a milder course of $\mathrm{LD}^{23}$ PTG is one of the glycogen targeting regulatory subunits of protein phosphatase 1 and it positively modulates the affinity of protein phosphatase 1 towards its substrates such as the glycogen synthase and phosphorylase. ${ }^{10}$ The laforin-malin complex was shown to negatively regulate the activity of PTG by promoting its degradation via the proteasome. ${ }^{10,24}$ As the loss of laforin or malin results in the genesis of Lafora bodies ${ }^{25,26}$ and suppression of PTG in an LD mouse model rescued, most of the disease symptoms and pathologies, ${ }^{27}$ it is reasonable to expect that the variant of PTG that associate with the milder phenotype of LD might show a diminished activity towards glycogen synthesis. Biochemical studies have shown that the LD-linked PTG variant indeed displayed decreased ability to promote the glycogen synthesis. ${ }^{23}$ Beyond glycogen synthesis, the laforin-malin complex is thought to be involved in other cellular pathways such as endoplasmic reticulum stress, ${ }^{28}$ clearance of misfolded proteins via the ubiquitin-proteasome pathway or autophagy $y^{11,29-31}$ and the heat shock response ${ }^{12}$ (see Table 1). It would be therefore important to check possible contributions of sequence variations in critical regulators of these pathways that interact with laforin and/or malin, and test their possible role as modifiers in LD. Given the emerging concept that phenotype variability in patients having the same gene mutation is dependent on the genetic background and environment, ${ }^{32}$ and studies have indeed established the contribution of genetic factors other than the disease-causing gene in the phenotype variability, ${ }^{33}$ it is imperative to extend such studies to LD as well. Such studies are likely to offer novel "druggable targets" that might help in delaying the onset or the severity of LD if not its treatment.

\section{ACKNOWLEDGEMENTS}

SS thanks the International Medical University, Kuala Lumpur, for the financial assistance. LD research in SG's laboratory is supported by research grants from the Department of Biotechnology and Department of Science \& Technology, Govt. of India. SG is a DAE-SRC Outstanding Research Investigator and the Gill-Joy Chair Professor.

1 Delgado-Escueta, A.V., Ganesh, S. \& Yamakawa, K. Advances in the genetics of progressive myoclonus epilepsy. Am. J. Med. Genet. 106, 129-138 (2001).

2 Ganesh, S., Puri, R., Singh, S., Mittal, S. \& Dubey, D. Recent advances in the molecular basis of Lafora's progressive myoclonus epilepsy. J. Hum. Genet. 51, 1-8 (2006).

3 Singh, S. \& Ganesh, S. Lafora progressive myoclonus epilepsy: a meta-analysis of reported mutations in the first decade following the discovery of the EPM2A and NHLRC1 genes. Hum. Mutat 30, 715-723 (2009).

4 Ramachandran, N., Girard, J.M., Turnbull, J. \& Minassian, B.A. The autosoma recessively inherited progressive myoclonus epilepsies and their genes. Epilepsia 50 29-36 (2009).

5 Chan, E.M., Omer, S., Ahmed, M. Bridges, L.R., Bennett, C., Scherer, S.W. \& Minassian, B.A. Progressive myoclonus epilepsy with polyglucosans (Lafora disease) evidence for a third locus. Neurology 63, 565-567 (2004).

6 Ganesh, S., Delgado-Escueta, A.V., Suzuki, T., Francheschetti, S., Riggio, C. \& Avanzini, G. et al. Genotype-phenotype correlations for EPM2A mutations in Lafora's progressive myoclonus epilepsy: exon 1 mutations associate with an early-onset cognitive deficit subphenotype. Hum. Mol. Genet. 11, 1263-1271 (2002).

7 Sakai, M., Austin, J., Witmer, F. \& Trueb, L. Studies in myoclonus epilepsy (Lafora body form). II. Polyglucosans in the systemic deposits of myoclonus epilepsy and in corpora amylacea. Neurology 20, 160-176 (1970).

8 Yokoi, S., Nakayama, H. \& Negishi, T. Biochemical studies on tissues from a patient with Lafora disease. Clin. Chim. Acta, 62, 415-423 (1975).

9 Busard, H.L., Renier, W.O., Gabreëls, F.J., Jaspar, H.H., Slooff, J.L., Janssen, A.J. \& Van Haelst, U.J. Lafora disease: a quantitative morphological and biochemical study of the cerebral cortex. Clin. Neuropathol. 6, 1-6 (1987).

10 Solaz-Fuster, M.C., Gimeno-Alcañiz, J.V., Ros, S., Fernandez-Sanchez, M.E., Garcia-Fojeda, B. \& Criado Garcia, O. et al. Regulation of glycogen synthesis by the 
laforin-malin complex is modulated by the AMP-activated protein kinase pathway. Hum. Mol. Genet. 17, 667-678 (2008).

11 Garyali, P., Siwach, P., Singh, P.K., Puri, R., Mittal, S. \& Sengupta, S. et al. The malinlaforin complex suppresses the cellular toxicity of misfolded proteins by promoting their degradation through the ubiquitin-proteasome system. Hum. Mol. Genet. 18, 688-700 (2009).

12 Sengupta, S., Badhwar, I., Upadhyay, M., Singh, S. \& Ganesh, S. Malin and laforin are essential components of a protein complex that protects cells from thermal stress. J. Cell Sci. 124, 2277-2286 (2011).

13 Singh, P.K., Singh, S. \& Ganesh, S. Laforin-malin complex negatively regulates glycogen synthesis by modulating the cellular glucose uptake via glucose transporters. Mol. Cell Biol 32, 652-663 (2012).

14 Gómez-Abad, C., Gómez-Garre, P., Gutiérrez-Delicado, E., Saygi, S., Michelucci, R. \& Tassinari, C.A. et al. Lafora disease due to EPM2B mutations: a clinical and genetic study. Neurology 64, 982-986 (2005).

15 Singh, S., Sethi, I., Francheschetti, S., Riggio, C., Avanzini, G. \& Yamakawa, K. et al. Novel NHLRC1 mutations and genotype-phenotype correlations in patients with Lafora's progressive myoclonic epilepsy. J. Med. Genet. 43, e48 (2006).

16 Annesi, G., Sofia, V., Gambardella, A., Candiano, I.C., Spadafora, P. \& Annesi, F. et al. A novel exon 1 mutation in a patient with atypical Lafora progressive myoclonus epilepsy seen as childhood-onset cognitive deficit. Epilepsia 45, 294-295 (2004).

17 Franceschetti, S., Gambardella, A., Canafoglia, L., Striano, P., Lohi, H. \& Gennaro, E. et al. Clinical and genetic findings in 26 Italian patients with Lafora disease. Epilepsia 47, 640-643 (2006).

18 Lesca, G., Boutry-Kryza, N., de Toffol, B., Milh, M., Steschenko, D. \& Lemesle-Martin, $M$. et al. Novel mutations in EPM2A and NHLRC1 widen the spectrum of Lafora disease. Epilepsia 51, 1691-1698 (2010).

19 Traoré, M., Landouré, G., Motley, W., Sangaré, M., Meilleur, K. \& Coulibaly, S. et al. Novel mutation in the NHLRC1 gene in a Malian family with a severe phenotype of Lafora disease. Neurogenetics 10, 319-323 (2009).

20 Baykan, B., Striano, P., Gianotti, S., Bebek, N., Gennaro, E., Gurses, C. \& Zara, F. Lateonset and slow-progressing Lafora disease in four siblings with EPM2B mutation. Epilepsia 46, 1695-1697 (2005).

21 Gomez-Abad, C., Afawi, Z., Korczyn, A.D., Misk, A., Shalev, S.A. \& Spiegel, R. et al. Founder effect with variable age at onset in Arab families with Lafora disease and EPM2A mutation. Epilepsia 48, 1011-1014 (2007).

22 Turnbull, J., Kumar, S., Ren, Z.P., Muralitharan, S., Naranian, T., Ackerley, C.A. \& Minassian, B.A. Lafora progressive myoclonus epilepsy: disease course homogeneity in a genetic isolate. J. Child Neurol. 23, 240-242 (2008).

23 Gómez-Garre, P., Gutiérrez-Delicado, E., Gómez-Abad, C., Morales-Corraliza, J., Villanueva, V.E. \& Rodríguez de Córdoba, S. et al. Hepatic disease as the first manifestation of progressive myoclonusepilepsy of Lafora. Neurology 68, 1369-1373 (2007).

24 Guerrero, R., Vernia, S., Sanz, R., Abreu-Rodríguez, I., Almaraz, C. \& García-Hoyos, M. et al. A PTG variant contributes to a milder phenotype in Lafora disease. PLoS One 6, e21294 (2011).

25 Vilchez, D., Ros, S., Cifuentes, D., Pujadas, L., Vallès, J. \& García-Fojeda, B. et al. Mechanism suppressing glycogen synthesis in neurons and its demise in progressive myoclonus epilepsy. Nat. Neurosci. 10, 1407-1413 (2007).

26 Ganesh, S., Delgado-Escueta, A.V., Sakamoto, T., Avila, M.R., Machado-Salas, J. \& Hoshii, Y. et al. Targeted disruption of the Epm2a gene causes formation of Lafora inclusion bodies, neurodegeneration, ataxia, myoclonus epilepsy and impaired behavioral response in mice. Hum. Mol. Genet. 11, 1251-1262 (2002).

27 DePaoli-Roach, A.A., Tagliabracci, V.S., Segvich, D.M., Meyer, C.M., Irimia, J.M. \& Roach, P.J. Genetic depletion of the malin E3 ubiquitin ligase in mice leads to Lafora bodies and the accumulation of insoluble laforin. J. Biol. Chem. 285, 25372-25381 (2010).
28 Turnbull, J., DePaoli-Roach, A.A., Zhao, X., Cortez, M.A., Pencea, N. \& Tiberia, E. et al. PTG depletion removes Lafora bodies and rescues the fatal epilepsy of Lafora disease. PLoS Genet. 7, e1002037 (2011).

29 Vernia, S., Rubio, T., Heredia, M., Rodríguez de Córdoba, S. \& Sanz, P. Increased endoplasmic reticulum stress and decreased proteasomal function in Lafora disease models lacking the phosphatase laforin. PLoS One 4, e5907 (2009).

30 Aguado, C., Sarkar, S., Korolchuk, V.I., Criado, O., Vernia, S. \& Boya, P. et al. Laforin, the most common protein mutated in Lafora disease, regulates autophagy. Hum. Mol. Genet. 19, 2867-2876 (2010).

31 Criado, O., Aguado, C., Gayarre, J., Duran-Trio, L., Garcia-Cabrero, A.M. \& Vernia, S. et al. Lafora bodies and neurological defects in malin-deficient mice correlate with impaired autophagy. Hum. Mol. Genet. 21, 1521-1533 (2011).

32 Puri, R., Suzuki, T., Yamakawa, K. \& Ganesh, S. Dysfunctions in endosomal-lysosomal and autophagy pathways underlie neuropathology in a mouse model for Lafora disease. Hum. Mol. Genet. 21, 175-184 (2012).

33 Nadeau, J.H. Modifier genes in mice and humans. Nat. Rev. Genet. 2, 165-174 (2001).

34 Cutting, G.R. Modifier genes in Mendelian disorders: the example of cystic fibrosis. Ann. N. Y. Acad. Sci. 1214, 57-69 (2010).

35 Ganesh, S., Tsurutani, N., Suzuki, T., Ueda, K., Agarwala, K.L. \& Osada, H. et al. The Lafora disease gene product laforin interacts with HIRIP5, a phylogenetically conserved protein containing a NifU-like domain. Hum. Mol. Genet. 12, 2359-2368 (2003).

36 lanzano, L., Zhao, X.C., Minassian, B.A. \& Scherer, S.W. Identification of a novel protein interacting with laforin, the EPM2a progressive myoclonus epilepsy gene product. Genomics 81, 579-587 (2003).

37 Lohi, H., Ianzano, L., Zhao, X.C., Chan, E.M., Turnbull, J. \& Scherer, S.W. et al. Novel glycogen synthase kinase 3 and ubiquitination pathways in progressive myoclonus epilepsy. Hum. Mol. Genet. 14, 2727-2736 (2005).

38 Wang, Y., Liu, Y., Wu, C., Zhang, H., Zheng, X. \& Zheng, Z. et al. Epm2a suppresses tumor growth in an immunocompromised host by inhibiting Wnt signaling. Cancer Cell 10, 179-190 (2006).

39 Cheng, A., Zhang, M., Gentry, M.S., Worby, C.A., Dixon, J.E. \& Saltiel, A.R. A role for AGL ubiquitination in the glycogen storage disorders of Lafora and Cori's disease. Genes Dev. 21, 2399-2409 (2007).

40 Moreno, D., Towler, M.C., Hardie, D.G., Knecht, E. \& Sanz, P. The laforin-malin complex, involved in Lafora disease, promotes the incorporation of K63-linked ubiquitin chains into AMP-activated protein kinase beta subunits. Mol. Biol. Cell. 21, 2578-2588 (2010).

41 Fernández-Sánchez, M.E., Criado-García, O., Heath, K.E., García-Fojeda, B., MedrañoFernández, I. \& Gomez-Garre, P. et al. Laforin, the dual-phosphatase responsible for Lafora disease, interacts with R5 (PTG), a regulatory subunit of protein phosphatase-1 that enhances glycogen accumulation. Hum. Mol. Genet 12, 3161-3171 (2003)

42 Worby, C.A., Gentry, M.S. \& Dixon, J.E. Malin decreases glycogen accumulation by promoting the degradation of protein targeting to glycogen (PTG). J. Biol. Chem. 283, 4069-4076 (2008).

43 Puri, R., Suzuki, T., Yamakawa, K. \& Ganesh, S. Hyperphosphorylation and aggregation of Tau in laforin-deficient mice, an animal model for Lafora disease. J. Biol. Chem. 284, 22657-22663 (2009).

44 Rao, S.N., Sharma, J., Maity, R. \& Jana, N.R. Co-chaperone CHIP stabilizes aggregate-prone malin, a ubiquitin ligase mutated in Lafora disease. J. Biol. Chem. 285, 1404-1413 (2010).

45 Sharma, J., Rao, S.N., Shankar, S.K., Satishchandra, P. \& Jana, N.R. Lafora disease ubiquitin ligase malin promotes proteasomal degradation of neuronatin and regulates glycogen synthesis. Neurobiol. Dis. 44, 133-141 (2011).

46 Sharma, J., Mulherkar, S., Mukherjee, D. \& Jana, N.R. Malin regulates Wnt signalling pathway through degradation of dishevelled 2. J. Biol. Chem. 287, 6830-6839 (2012). 